\title{
TERT promoter wild-type glioblastomas show distinct clinical features and frequent PI3K pathway mutations
}

\author{
Erik A. Williams ${ }^{1,2}$, Julie J. Miller ${ }^{1,3}$, Shilpa S. Tummala1, Tristan Penson ${ }^{1}$, A. John lafrate ${ }^{2}$, Tareq A. Juratli ${ }^{1+}$
} and Daniel P. Cahill ${ }^{1 *+}$

\begin{abstract}
TERT promoter (TERTp) mutations are found in the majority of World Health Organization (WHO) grade IV adult IDH wild-type glioblastoma (IDH-wt GBM). Here, we characterized the subset of IDH-wt GBMs that do not have TERTp mutations. In a cohort of 121 adult grade IV gliomas, we identified 109 IDH-wt GBMs, after excluding 11 IDH-mutant cases and one H3F3A -mutant case. Within the IDH-wt cases, 16 cases (14.7\%) were TERTp wild-type (TERTp-wt). None of the 16 had BRAF V600E or H3F3A G34 hotspot mutations. When compared to TERTp mutants, patients with TERTp-wt GBMs, were significantly younger at first diagnosis (53.2 years vs. 60.7 years, $p=0.0096)$, and were more frequently found to have cerebellar location ( $p=0.0027)$. Notably, 9 of 16 (56\%) of TERTp-wt GBMs contained a PIK3CA or PIK3R1 mutation, while only 16/93 (17\%) of TERTp-mutant GBMs harbored these alterations ( $p=0.0018$ ). As expected, 8/16 (50\%) of TERTp-wt GBMs harbored mutations in the BAF complex gene family (ATRX, SMARCA4, SMARCB1, and ARID1A), compared with only 8/93 (9\%) of TERTp-mutant GBMs $(p=0.0003)$. Mutations in BAF complex and PI3K pathway genes co-occurred more frequently in TERTp-wt GBMs $(p=0.0002)$, an association that has been observed in other cancers, suggesting a functional interaction indicative of a distinct pathway of gliomagenesis. Overall, our finding highlights heterogeneity within WHO-defined IDH wild-type GBMs and enrichment of the TERTp-wt subset for BAF/PI3K-altered tumors, potentially comprising a distinct clinical subtype of gliomas.
\end{abstract}

Keywords: Glioma, PI3K pathway, TERT promoter, IDH1, H3F3A, BAF complex, Cerebellum

\section{Introduction}

Glioblastoma (GBM) is the most frequent and deadly primary brain tumor, accounting for approximately $45-50 \%$ of all primary malignant brain tumors $[17,18]$. GBM is a heterogeneous entity, with a wide mutational spectrum. There has been an ever-increasing focus on molecular classification in GBM, to develop insights into the biology of this tumor and to subsequently improve diagnosis and treatment.

To emphasize the importance of molecular markers, the 2016 World Health Organization (WHO) revised neuropathological criteria identifies three categories of grade IV diffuse glioma. Two categories of GBM arise

\footnotetext{
* Correspondence: cahill@mgh.harvard.edu

'Tareq A Juratli and Daniel P Cahill contributed equally to this work.

${ }^{1}$ Translational Neuro-Oncology Laboratory, Department of Neurosurgery, Massachusetts General Hospital Cancer Center, Harvard Medical School, 55 Fruit street, Boston, MA, USA

Full list of author information is available at the end of the article
}

based on clustered genetic alterations, histologic variants, and clinical data [15], $I D H$ wild-type and $I D H$ mutant. An additional category of H3F3A K27 M mutant midline glioma has been designated grade IV, due the often poor prognosis of patients with these tumors. While $I D H$ and $H 3 F 3 A$ mutations identify gliomas with a distinct molecular origin, the remaining $I D H$ wild-type subgroup of GBM, as it is defined currently, still contains significant heterogeneity. Emerging evidence indicates that TERT promoter (TERTp) mutations, which are common in these tumors, could additionally be useful clinically to classify $I D H$ wild-type GBMs into subgroups with specific clinical courses [7, 12].

Here, we evaluated TERTp wild-type (TERTp-wt) GBMs to compare them to their TERTp mutant counterpart GBMs. We performed sequencing on a broad panel of genes and evaluated for the presence of fusions 
in a cohort of GBMs, to evaluate the mutational profile of TERTp-wt GBMs. In addition, we examined the clinical characteristics of this group.

\section{Material and methods}

The study was reviewed and approved by the human subjects' institutional review boards of the Dana-Farber Cancer Institute and Massachusetts General Hospital (P10-454) and complied with HIPAA guidelines. We retrospectively reviewed the genomic database at our institution for adult GBM cases submitted for genotyping using the SNaPshot panel version 2. Demographic, treatment and follow-up data were retrospectively collected.

\section{SNaPshot next generation sequencing archer $^{\circledR}$ FusionPlex ${ }^{\circledast}$}

Specimens were subjected to genomic analysis utilizing $\mathrm{SNaPshot}^{6}$, a hybrid capture based method for single nucleotide variant (SNV) and insertion/deletion (indel) detection in tumor DNA. SNaPshot targets 108 genetic loci frequently mutated in 15 cancer genes, including TERT promoter, IDH1/2, TP53, ATRX, PIK3CA, PIK3R1, $N F 1$, and STAG2. The detailed list of all genes included in the SNaPshot v2 panel is shown in Additional file 1.

\section{Archer $^{\circledast}$ FusionPlex $^{\circledR}$}

Extracted tumor RNA were interrogated for fusions by the Archer ${ }^{\oplus}$ FusionPlex ${ }^{\circ}$ Solid Tumor (AK0034) kit [25]. This technology utilizes an anchored multiplex polymerase chain reaction (AMP) technique that detects gene rearrangements in a fusion partner agnostic manner. FASTQ data analysis, including fusion calling, was performed by ArcherDx Analysis software v5.0.6 using default parameters. The detailed list of all genes included in Archer $^{\circ}$ FusionPlex ${ }^{\circ}$ is shown in Additional file 1.

\section{MGMT promoter methylation}

DNA was extracted from frozen tumor tissue and subjected to bisulfite treatment. Two separate methylation-specific PCR reactions were performed, one using primers specific for methylated MGMT promoter sequences, and a second using PCR primers specific for unmethylated MGMT promoter sequences [8].

\section{ATRX immunohistochemistry methods}

ATRX immunohistochemistry was preformed using ATRX Cat \# BSB-3295 from Bio SB. RTU (ready to use) pretreatment ER2 (EDTA ph 9.0) for $15 \mathrm{~min}$. The clone BSB-108 was used, as previously reported [21].

\section{Statistical analysis}

The statistical association of TERTp-wt GBM with other factors, including age, sex, other genomic alterations, and location of tumor, were analyzed using the Fisher exact test. The association of TERTp-wt GBM with ATRX immunohistochemistry, MGMT promoter methylation status, and presence of fusion gene by solid fusion panel were each also evaluated. Cases with unavailable molecular or IHC data were excluded from the final correlation analysis.

The data were analyzed using the Fisher exact test. Description of overall survival (OS) was estimated by the Kaplan-Meier product limit method. A two-tailed $P$ value of $<0.05$ was considered to be statistically significant.

\section{Results}

\section{Patient demographic and tumor characteristics}

We identified 121 adult GBM cases with available molecular and immunohistochemistry data between 2016 and 2018 (Additional file 2). We excluded histologic GBMs containing IDH R132 and H3F3A mutations from statistical analyses ( $n=11$ and $n=1$, respectively), for the reasons noted above [15].

Within this cohort $(n=109)$, the average age of patients was 60 years (range 18-84 years). Genetic alterations in the TERT gene were detected in 93 tumors; 92 were TERTp mutant (84.4\%), and an additional case had a TERT-SUB fusion. The remaining 16 patients (14.7\%) had TERTp-wt GBM (Fig. 1 and Additional file 2). The average age of patients with TERTp-wt GBMs was 53.2 years, which was significantly younger than the average age of their counterparts with TERTp mutant GBMs (60.7 years, $p=0.0096)$, and significantly older than the average age of patients with $I D H$ mutant GBMs (38.6 years, $p=0.0041$ ).

Across the cohort of $I D H$-wt GBM, the male to female ratio was 1.66. TERTp-wt GBMs did manifest a numerically higher proportion of male patients $(13 / 16,87.5 \%)$, compared with 55/93 male patients (59\%) with TERTp mutant GBMs, but this difference was not statically significant ( $p=0.103)$.

We examined the location of the primary tumor presentation. In the TERTp-wt group, the primary tumors were mainly found in a supratentorial (13) and thalamic/midline location (1), but also in a cerebellar site (3 cases). In contrast, in the TERTp mutant group, the tumors were exclusively located supratentorially (91) or thalamic/midline (2), with none found in the cerebellum. Consequently, a significant correlation between TERTp-wt status and cerebellar location $(p=0.0027)$ was observed. Of note, one of the cerebellar GBMs occurred in a patient with a NF1 germline mutation (Neurofibromatosis type 1 ).

The median time of follow-up in surviving patients was 189 days for the TERTp-wt group and 246 days for the TERTp mutant group. Due to the short follow-up time, survival analyses may be underpowered to detect differences. Nonetheless, no detectable difference in survival was observed between the two groups $(p=0.74)$. 


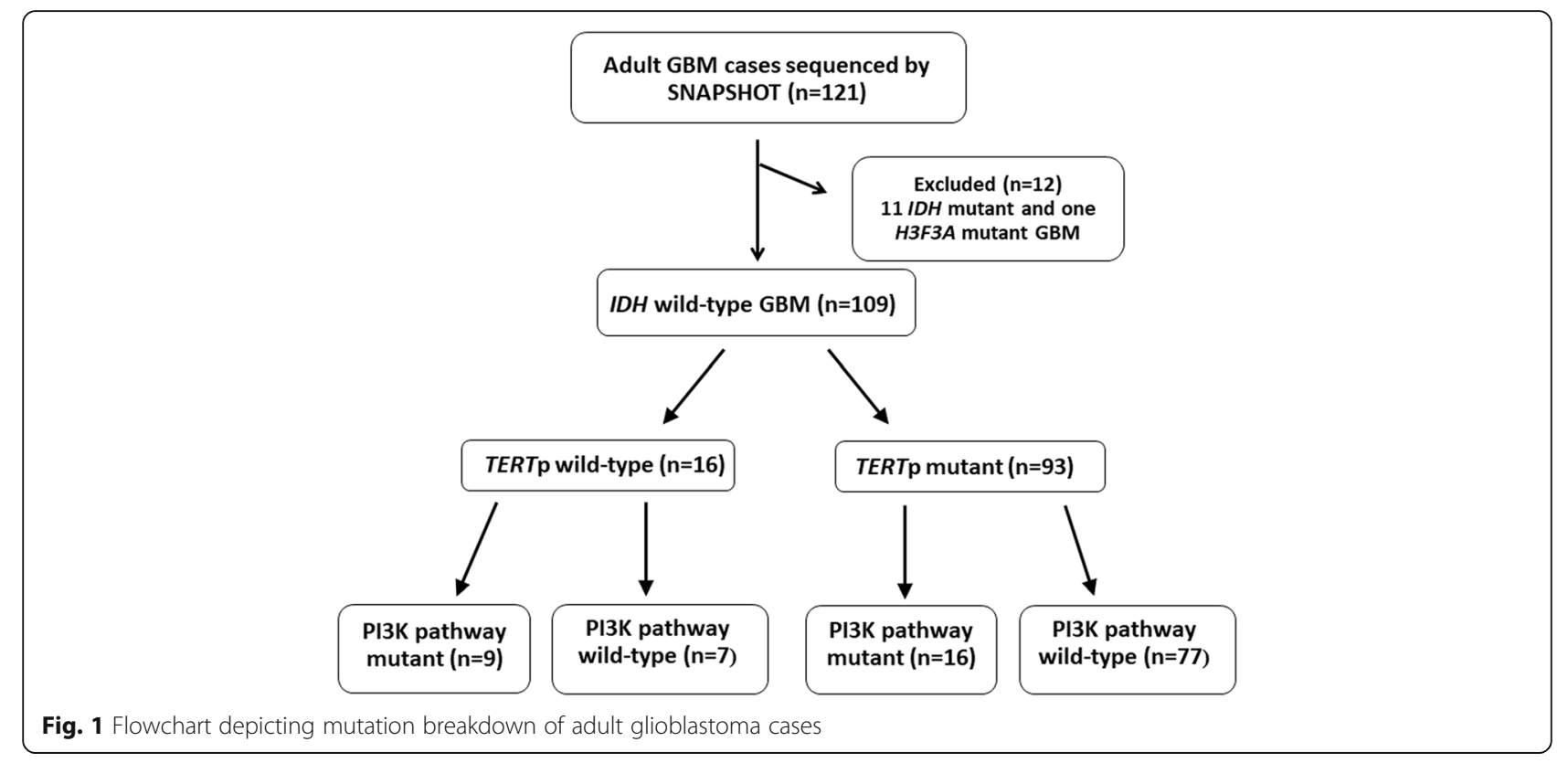

\section{Genetic and epigenetic correlation}

We examined genetic and epigenetic correlations between TERTp-wt versus mutant tumors. Four TERTp mutant cases were found to harbor a hotspot $B R A F$ V600E mutation, which is characteristic of epithelioid GBM [14]. NF1 mutations were more commonly seen in TERTp-wt GBMs (6/16, 37.5\%), in comparison with 18/ 93 (19\%) in the TERTp mutant GBM cohort, however, this was not a statistically significant difference ( $p=$ 0.11 ). Also, we did not observe a significant difference in MGMT promoter methylation status in the TERTp-wt group vs. the mutant group $(7 / 14$ vs. $36 / 90, p=0.56)$.

Activating alterations in the PI3K pathway (mainly PIK3CA or PIK3R1) were detected in 25 out of 109 cases in the cohort (23\%) (Additional file 3). Interestingly, we observed a strong correlation between TERTp-wt status and mutations targeting the PI3K pathway: 9/16 (56\%) of TERTp-wt GBMs contained a PI3K pathway alteration, while only 16/93 (17\%) of mutant GBMs harbored these alterations $(p=0.0018)$ (Fig. 1). Furthermore, we detected an inverse correlation between PIK3CA/PIK3R1 and EGFR alterations. Only $2 / 25$ cases $(8 \%)$ with a PI3K pathway alteration had an EGFR mutation or EGFRvIII, whereas 38/82 of PI3K wild-type GBM had an EGFR alteration (46.3\%, $p$ $=0.0003)$.

Moreover, as expected, ATRX mutations were detected by sequencing in 6/16 (37.5\%) TERTp-wt GBMs, while only 6/93 (6.5\%) of TERTp mutant GBMs had an ATRX mutation. Consequently, this manifested as a significant correlation between TERTp-wt status and ATRX mutation $(p=0.0022)$. Of note, our workflow for assigning mutation was highly sensitive, leading to potential false positive assignments of $A T R X$ candidate alterations that may not functionally inactivate the protein product. The further assessment of ATRX loss-of-expression using immunohistochemistry revealed a similarly significant result: 4/13 (31\%) of TERTp-wt GBMs had ATRX loss vs. 0/80 mutant GBMs $(p=0.0002)$ (Fig. 2).

Finally, we noted that $8 / 16$ (50\%) of TERTp-wt GBMs harbored mutations in the BAF complex gene family (SMARCA4, SMARCB1, ATRX, and ARID1A), compared with only $8 / 93$ of TERTp mutant GBMs $(p=0.0002)$. Given the role of $A T R X$ in telomere maintenance, mutations in either group (ATRX vs SWI/SNF) may be unrelated. Nevertheless, we found that this association remained significant when excluding ATRX (3/16 (18.8\%) of TERTp-wt GBMs harboring mutations compared with only 2/93 of TERTp mutant GBMs, $p=0.022$ ). When combined with our analyses above, we detected a significant difference in co-occurrence between mutations in the BAF complex and PI3K pathway genes by comparing the TERTp-wt $(n=5 / 16)$ and TERTp mutant groups $(n=1 / 93$, $p=0.0002$ ) (Fig. 3).

\section{Discussion}

The WHO 2016 established an IDH wild-type subgroup of GBM, comprising the majority of adult grade IV gliomas, yet, this diagnostic grouping still contains significant heterogeneity. In an effort to better sub-classify $I D H$-wt GBMs, we used a broad panel of genes to genotype a large cohort of these neoplasms. In our analyses, we show that the TERTp-wt subgroup of $I D H$-wt GBM contains a distinct clinical and molecular profile.

Our findings should be interpreted in the context of extensive recent work studying adult high-grade gliomas. Over the last several years, a strong relationship has been 


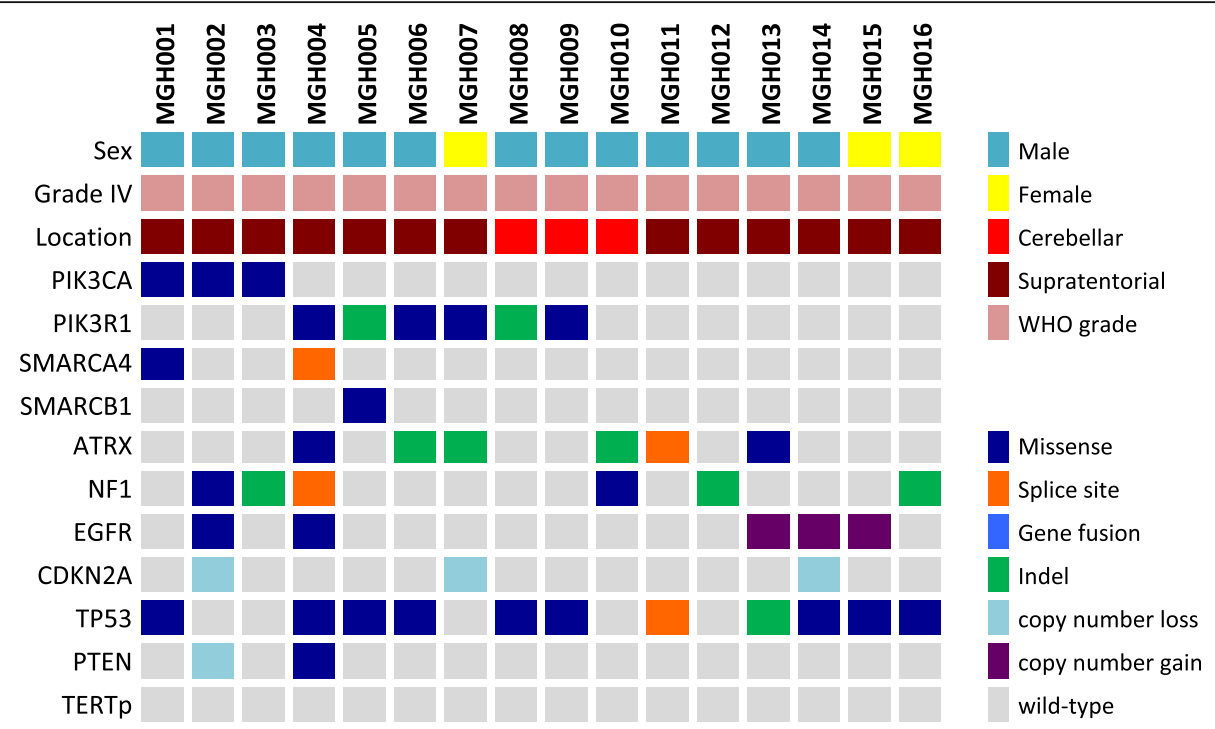

Fig. 2 Summary of clinical features and molecular alterations in TERTp-wt glioblastoma

demonstrated between mutational status and clinical, radiological, and molecular characteristics in adult diffuse gliomas [1, 2, 7]. Recently, Eckel-Passow et al. identified five main glioma molecular groups based on three alterations: $1 \mathrm{p} / 19 \mathrm{q}$ co-deletion, and TERTp and $I D H$ mutations. The groups had different ages of onset, survival, and associations with germline variants [7]. In addition, Aibaidula et al. specifically examined the adult $I D H$ wild-type lower-grade gliomas, demonstrating significant heterogeneity within this group, with differences in prognosis based on further molecular classification by biomarkers such as
TERTp mutation, EGFR amplification, H3F3A mutation, and $M Y B$ amplification [1]. Focusing on GBM, Arita et al. highlighted the importance of TERTp mutation, $I D H \mathrm{mu}$ tation, and MGMT promoter methylation status on prognosis [2]. Furthermore, Stichel et al. demonstrated the potential of EGFR amplification, combined chromosome 7 gain and chromosome 10 loss, and TERTp mutations for classifying $I D H$ wild-type GBM [24].

TERTp mutant GBMs show increased telomerase activation due to the increased TERT expression. In comparison, it is well-established that $I D H$ mutant astrocytic gliomas a TERTp wild-type GBM
$(\mathrm{n}=16)$

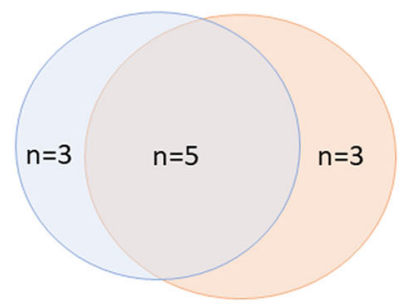

b

TERTp mutant GBM $(n=93)$

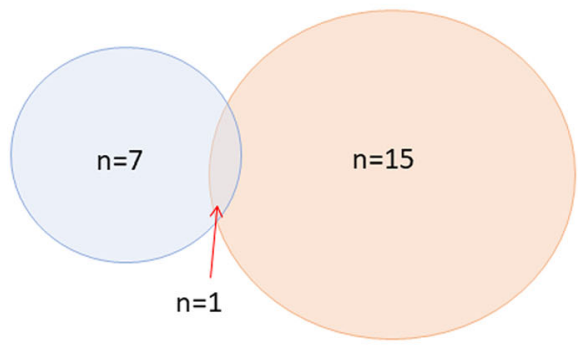

PI3K pathway mutations

BAF complex member mutations

Fig. 3 Venn diagram depicting BAF complex mutation (SMARCA4, SMARCB1, ATRX, or ARIDIA) and PI3K mutations relationship in TERT p-wt (a) and TERT p mutant (b) glioblastomas (GBM). Cases negative for both BAF complex and PI3K mutations amounted to $n=5$ and $n=70$, respectively 
often display the characteristic phenotype termed "alternative lengthening of telomeres" or ALT, associated with mutations in $\operatorname{ATRX}[10,11,13]$. Another study that attempted to further sub-classify the 5 integrated WHO glioma groups by $A T R X$ and TERT promoter status showed that ATRX alterations were enriched in TERTp-wt GBM [19]. A further study of the TERTp-wt subgroup by Diplas et al. identified SMARCAL1 as an additional mechanism of telomere maintenance within this subgroup [6].

In agreement with prior studies, we observed that $T E R T \mathrm{p}$-wt patients are significantly younger than their TERTp mutant counterparts [7] (Additional file 4). In addition, we identified a significantly higher rate of cerebellar GBM in the TERTp-wt compared with the TERTp mutant patients. Our finding is consistent with prior studies that have shown that cerebellar GBMs occur in patients that are younger than patients with supratentorial GBMs, and have decreased frequency in TERTp mutations and more frequent NF1 mutations [16, 20]. Taken together with our findings, these data support the proposal that cerebellar GBMs may comprise a distinct subclass of tumor, which may arise via an alternative molecular etiology when compared to supratentorial TERTp mutant GBM.

PI3K pathway alterations are frequently detected in gliomas, most commonly in grade IV lesions [4, 9]. Our data demonstrate that TERTp-wt GBMs are significantly enriched for PI3K pathway mutations compared with TERTp mutant GBM. Moreover, mutations in ARID1A and other components of the SWI/SNF chromatin remodeling complex (collectively known as the BAF complex), have been previously reported to be frequent in various cancer types (e.g. endometriosis-associated ovarian cancers, endometrial cancers and non-gynecological tumors) $[3,5,22$, 23]. Interestingly, in these cancers, alterations of gene encoding for components of the BAF complex frequently co-occur with activating mutations in PIK3CA [3, 23]. It has been additionally reported that dysregulation of the PI3K signaling pathway and loss of function of ARID1A may have a combination effect on tumor development [5, 22]. We speculate that this association may extend to a specific subset of gliomas, namely TERTp-wt GBM cases, which we find are enriched for BAF complex alterations and activating mutations in genes within the PI3K pathway. Following the logic of WHO 2016 classification, our findings suggest the potential definition of a molecular subtype of high-grade glioma, with implications for the utilization of targeted therapy in these patients [22].

\section{Conclusions}

In conclusion, this study identifies frequent PI3K pathway and BAF complex genetic alterations as co-occurring hallmarks of TERTp-wt GBM, potentially reflecting a unique molecular etiology of these tumors. If further validated, these findings may have significant implications for the sub-classification of $I D H$-wt GBM. Optimal management of these patients remains to be defined, but at a minimum, our data suggest that TERTp-mutant and TERTp-wt GBMs should be analyzed separately in future clinical studies, as they likely comprise distinct subclasses of neoplastic disease.

\section{Additional files}

Additional file 1: A detailed list of all genes included in the SNaPshot v2 panel. (DOCX 14 kb)

Additional file 2: A table including patients' and tumor characteristics. (XLSX $20 \mathrm{~kb}$ )

Additional file 3: A table listing all detected PI3K alterations in the cohort. The majority of alterations were reported in COSMIC (https:// cancer.sanger.ac.uk/cosmic/browse/genome) and/or occured at hospot locations in TumorPortal (http://www.tumorportal.org/). Reference human transcripts used: ENST00000263967.3 (PIK3CA) and ENST00000521381.1 (PIK3R1). (XLSX $11 \mathrm{~kb})$

Additional file 4: Age distribution according to TERTp mutations. (TIF $462 \mathrm{~kb}$ )

\section{Funding}

This work is supported by U.S. NIH T32 CA009216 (to Dr. E. Williams) the Deutsche Forschungsgemeinschaft (DFG, German Research Foundation) - Projektnummer: 401837860 (to Dr. T. Juratli) and by the Burroughs Wellcome Fund Career Award (to Dr. D. Cahill).

\section{Authors' contributions}

EAW carried out the research studies. EWA and AJl performed the sequence alignment. SST and TP participated in the sequence alignment. EAW, JJM, TAJ, and DPC designed the study. EAW and TAJ performed the statistical analysis. EAW, TAJ and DPC drafted the manuscript. All authors read and approved the final manuscript.

\section{Competing interests}

The authors declare that they have no competing interest.

\section{Publisher's Note}

Springer Nature remains neutral with regard to jurisdictional claims in published maps and institutional affiliations.

\section{Author details}

${ }^{1}$ Translational Neuro-Oncology Laboratory, Department of Neurosurgery, Massachusetts General Hospital Cancer Center, Harvard Medical School, 55 Fruit street, Boston, MA, USA. ²Department of Pathology, Massachusetts General Hospital, Harvard Medical School, Boston, MA, USA. ${ }^{3}$ Stephen E. and Catherine Pappas Center for Neuro-Oncology, Department of Neurology, Massachusetts General Hospital, Harvard Medical School, 55 Fruit Street, Boston, MA 02114, USA.

Received: 13 September 2018 Accepted: 5 October 2018

Published online: 17 October 2018

References

1. Aibaidula A, Chan AK, Shi Z, Li Y, Zhang R, Yang R et al (2017) Adult IDH wild-type lower-grade gliomas should be further stratified. Neuro-Oncology 19:1327-1337

2. Arita H, Yamasaki K, Matsushita Y, Nakamura T, Shimokawa A, Takami H et al (2016) A combination of TERT promoter mutation and MGMT methylation status predicts clinically relevant subgroups of newly diagnosed glioblastomas. Acta Neuropathol Commun 4:79

3. Bosse T, Ter Haar NT, Seeber LM, v Diest PJ, Hes FJ, Vasen HF et al (2013) Loss of ARID1A expression and its relationship with PI3K-Akt pathway 
alterations, TP53 and microsatellite instability in endometrial cancer. Mod Pathol 26:1525-1535

4. Broderick DK, Di C, Parrett TJ, Samuels YR, Cummins JM, McLendon RE et al (2004) Mutations of PIK3CA in anaplastic oligodendrogliomas, high-grade astrocytomas, and medulloblastomas. Cancer Res 64:5048-5050

5. Chandler RL, Damrauer JS, Raab JR, Schisler JC, Wilkerson MD, Didion JP et al (2015) Coexistent ARID1A-PIK3CA mutations promote ovarian clear-cell tumorigenesis through pro-tumorigenic inflammatory cytokine signalling. Nat Commun 6:6118

6. Diplas BH, He X, Brosnan-cashman JA, Liu H, Chen LH, Wang Z et al (2018) The genomic landscape of TERT promoter wildtype-IDH wildtype glioblastoma. Nat Commun 9:2087

7. Eckel-Passow JE, Lachance DH, Molinaro AM, Walsh KM, Decker PA, Sicotte $\mathrm{H}$ et al (2015) Glioma groups based on 1p/19q, IDH, and TERT promoter mutations in tumors. N Engl J Med 372:2499-2508

8. Esteller M, Garcia-Foncillas J, Andion E, Goodman SN, Hidalgo O, Vanaclocha $V$ et al (2000) Inactivation of the DNA-repair gene MGMT and the clinical response of gliomas to alkylating agents. N Engl J Med 343: 1350-1354

9. Gallia GL, Rand V, Siu IM, Eberhart CG, James CD, Marie SK et al (2006) PIK3CA gene mutations in pediatric and adult glioblastoma multiforme. Mo Cancer Res 4:709-714

10. Jiao Y, Killela PJ, Reitman ZJ, Rasheed AB, Heaphy CM, de Wilde RF et al (2012) Frequent ATRX, CIC, FUBP1 and IDH1 mutations refine the classification of malignant gliomas. Oncotarget 3:709-722

11. Kannan K, Inagaki A, Silber J, Gorovets D, Zhang J, Kastenhuber ER et al (2012) Whole-exome sequencing identifies ATRX mutation as a key molecular determinant in lower-grade glioma. Oncotarget 3:1194-1203

12. Killela PJ, Pirozzi CJ, Healy P, Reitman ZJ, Lipp E, Rasheed BA et al (2014) Mutations in IDH1, IDH2, and in the TERT promoter define clinically distinct subgroups of adult malignant gliomas. Oncotarget 5: 1515-1525

13. Killela PJ, Reitman ZJ, Jiao Y, Bettegowda C, Agrawal N, Diaz LA Jr et al (2013) TERT promoter mutations occur frequently in gliomas and a subset of tumors derived from cells with low rates of self-renewal. Proc Natl Acad Sci U S A 110:6021-6026

14. Kleinschmidt-DeMasters BK, Aisner DL, Birks DK, Foreman NK (2013) Epithelioid GBMs show a high percentage of BRAF V600E mutation. Am J Surg Pathol 37:685-698

15. Louis DN, Perry A, Reifenberger G, von Deimling A, Figarella-Branger D, Cavenee WK et al (2016) The 2016 World Health Organization classification of tumors of the central nervous system: a summary. Acta Neuropathol 131: 803-820

16. Nomura M, Mukasa A, Nagae G, Yamamoto S, Tatsuno K, Ueda H et al (2017) Distinct molecular profile of diffuse cerebellar gliomas. Acta Neuropathol 134:941-956

17. Ohgaki H, Kleihues P (2005) Population-based studies on incidence, survival rates, and genetic alterations in astrocytic and oligodendroglial gliomas. J Neuropathol Exp Neurol 64:479-489

18. Ostrom QT, Gittleman H, Liao P, Rouse C, Chen Y, Dowling J et al (2014) CBTRUS statistical report: primary brain and central nervous system tumors diagnosed in the United States in 2007-2011. Neuro Oncol 16(Suppl 4):iv1-i63

19. Pekmezci M, Rice T, Molinaro AM, Walsh KM, Decker PA, Hansen H et al (2017) Adult infiltrating gliomas with WHO 2016 integrated diagnosis: additional prognostic roles of ATRX and TERT. Acta Neuropathol 133:1001-1016

20. Picart T, Barritault M, Berthillier J, Meyronet D, Vasiljevic A, Frappaz D et al (2018) Characteristics of cerebellar glioblastomas in adults. J Neuro-Oncol 136:555-563

21. Reuss DE, Sahm F, Schrimpf D, Wiestler B, Capper D, Koelsche C et al (2015) ATRX and IDH1-R132H immunohistochemistry with subsequent copy number analysis and IDH sequencing as a basis for an "integrated" diagnostic approach for adult astrocytoma, oligodendroglioma and glioblastoma. Acta Neuropathol 129:133-146

22. Samartzis EP, Gutsche K, Dedes K, Fink D, Stucki M, Imesch P (2014) Loss of ARID1A expression sensitizes cancer cells to PI3K- and AKT-inhibition. Oncotarget 5:5295-5303

23. Samartzis EP, Noske A, Dedes KJ, Fink D, Imesch P (2013) ARID1A mutations and PI3K AKT pathway alterations in endometriosis and endometriosisassociated ovarian carcinomas. Int J Mol Sci 14:18824-18849
24. Stichel D, Ebrahimi A, Reuss D, Schrimpf D, Ono T, Shirahata M et al (2018) Distribution of EGFR amplification, combined chromosome 7 gain and chromosome 10 loss, and TERT promoter mutation in brain tumors and their potential for the reclassification of IDHwt astrocytoma to glioblastoma. Acta Neuropathol. https://doi.org/10.1007/s00401-018-1905-0

25. Zheng Z, Liebers M, Zhelyazkova B, Cao Y, Panditi D, Lynch K et al (2014) Anchored multiplex PCR for targeted next-generation sequencing. Nat Med 20:1479-1484

Ready to submit your research? Choose BMC and benefit from

- fast, convenient online submission

- thorough peer review by experienced researchers in your field

- rapid publication on acceptance

- support for research data, including large and complex data types

- gold Open Access which fosters wider collaboration and increased citations

- maximum visibility for your research: over 100M website views per year

At BMC, research is always in progress.

Learn more biomedcentral.com/submissions 